Ann. Zootech., I966, 15 (I), 5-I4.

\title{
EFFETS DE DIFFERENTS MODES DE REFROIDISSEMENT SUR LA MOTILITÉ DU SPERME DE TAUREAU APRÈS DÉCONGÉLATION
}

\author{
M. GOFFALX \\ avec la collaboration technique de J. C. Tourneur \\ Centre national de Recherches zootechniques, \\ Laboratoire de Physiologie de la Reproduction, 78 -Jouy-en-Josas \\ Union nationale des Coopératives d'Élerage et d'Insémination artificielle
}

\section{SOMMAIRE}

La présente expérimentation a été réalisée en vue d'étudier les effets, sur le pourcentage de spermatozoïdes mobiles après décongélation, de certaines particularités du diagramme de congélation, se situant immédiatement avant et après la formation des premiers cristaux : surfusion, réchauffement dû à la libération de la chaleur de fusion et maintien pendant un temps plus ou moins long d'une température constante ì la suite de ce réchauffement (plateau de début de cristallisation).

-.. Quarante-deux éjaculats provenant de 6 taureaux ont été dilués dans um milieu lait écrémé-fructose-glycérol, conditionnés en ampoules de verre de $1,2 \mathrm{ml}$ et congelés selon 7 programmes présentant, diversement associés, les caractères ci-dessus indiqués.

- Aucune différence n’a été observée entre les pourcentages de spermatozoìdes mobiles des échantillons soumis à des programmes de refroidissement présentant ou non les particularités concomitantes de surfusion et de réchauflement.

- Le maintien, pendant 30 secondes, d'une tenpérature constante (plateau) au début de la cristallisation, s'est montré plus favorable $(\mathrm{P}<\mathrm{o}$,o I) du'un plateau analogue de 5 minutes, ce dernier étant lui-même supérieur à un refroidissement lent de $0,8^{\circ} \mathrm{C} / \mathrm{mn}$ poursuivi pendant le même temps.

Les échantillons témoins, congelés selon un programme de refroidissement relativement rapide $\left(24^{\circ} \mathrm{C} / \mathrm{mn}\right.$ avant cristallisation) ne présentant ni surfusion ni réchauffement, ont révélé des pourcentages de spermatozoïdes mobiles ne différant pas significativenent de ceus relatifs au meilleur traitement.

- Ces résultats suggèrent que des refroidissements initiaux lents, accélérés insuffisamment ou à un moment trop éloigné de la formation des premiers cristaux, peuvent se révéler moins favorables à la survie des spermatozoïdes de taureaux que certains refroidissements plus rapides.

\section{INTRODUCTION}

La diversité des diagrammes de congélation de sperme employés actuellement en insémination artificielle bovine, illustre les contradictions exprimées dans la bibliographie. Malgré les différences constatées dans la façon de mesurer les tem- 
pératures (à l'extérieur ou à l'intérieur de l'échantillon) et dans la durée du refroidissement initial (poursuivi jusqu'à $-\mathrm{I}^{\circ} \mathrm{C},-\mathrm{I}^{\circ} \mathrm{C}$ ou $-20^{\circ} \mathrm{C}$ ), 1'ensemble des publications de POLGE et LoveI,OCK (I952), MIL, I, et VAN I) EMark (I954), O'DEIL, et al. (I958), BRUEMMER et al. (I963) se montre favorable à un abaissement thermique lent $\left(\mathrm{I}^{\circ} \mathrm{C}\right.$ à $\left.5^{\circ} \mathrm{C} / \mathrm{mn}\right)$ au début des opérations de congélation. Én revanche, différents auteurs concluent à l'égalité ou à la supériorité des effets d'un refroidissement initial plus rapide $\left(\mathrm{I}^{\circ} \mathrm{C}\right.$ à $\left.46^{\circ} \mathrm{C} / \mathrm{mn}\right)$ obtenu par l'introduction des échantillons dans des bains d'alcool successifs (BIALy et SMITH, I957; KrNNELY et al., I960) au milieu de fragments de glace carbonique (POLGE et JACOBSEN, I959; BERGFELD, I963) ou dans la partie gazeuse d'un récipient renfermant de l'azote liquide (MarTiG et DICKEY, I 963 ).

L'étude des diagrammes température-temps obtenus, au cours des opérations de congélation, à l'aide de thermocouples placés au milieu des échantillons, montre qu'à un refroidissement initial qualifié sommairement de "lent " peuvent correspondre des diagrammes en fait assez différents. Selon la température choisie pour intensifier l'apport de froid, selon les caractéristiques physiques du réfrigérant et de l'échantillon (qualité du contact, chaleurs spécifiques, conductibilité, épaisseur de paroi, volume, forme), on obtient des diagrammes présentant, après une chute de température de même pente avant cristallisation, une surfusion plus ou moins profonde, un réchauffement plus ou moins net et durable après formation des premiers cristaux et un abaissement thermique consécutif plus ou moins rapide.

La diversité de ces diagrammes a semblé pouvoir rendre compte en partie des contradictions relevées dans la bibliographie : certains refroidissements lents pourraient associer des particularités qui les rendraient moins favorables que tels refroidissements rapides, alors que d'autres refroidissements lents leur seraient au contraire supérieurs.

Cette expérimentation a été entreprise pour étudier les effets des caractères associés de surfusion et de réchauffement et ceux du maintien pendant un temps plus ou moins long d'une température constante à la suite de ce réchauffement (plateau de début de cristallisation). Une expérimentation précédente (GoFfaux, I964) n'ayant pas reconnu d'effet à la durée du plateau de début de cristallisation dans les limites de I minute et 3 minutes 30 secondes, des écarts plus sensibles entre traitements ont été choisis. L'effet d'un refroidissement lent après formation des premiers cristaux (" plateau " incliné de $0,8 \circ \mathrm{C} / \mathrm{mn}$ ) a été également étudié.

\title{
MATÉRIEL, ET MÉTHODES
}

\begin{abstract}
Quarante-deux éjaculats provenant de 6 taureaux prélevés une fois par semaine ont été dilués dans le milieu lait écrémé-fructose-glycérol-antibiotiques emprunté à ALMQUIST et décrit précédemment (GoFFAUX, I963). Après réfrigération à $+4^{\circ} \mathrm{C}$ en 2 heures trente minutes et glycérolisation en 30 minutes par 3 additions à I 5 minutes d'intervalle du dilueur glycérolisé, la semence a subi un temps d'équilibration de 4 heures. Le nombre total de spermatozoïdes a été ajusté à $3^{\circ}$ millions par millilitre de sperme dilué pour cha(jue éjaculat. La semence a été conditionnée dans des ampoules de verre de $1,2 \mathrm{ml}$ à paroi épaisse de $\mathrm{I} \mathrm{mm}$, à raison de $\mathrm{I}$ inl de sperme dilué par ampoule.

Les congélations ont été réalisées par immersion de ces ampoules dans des bains successifs d'alcool maintenus à des températures définies. Sept types de diagranmes ont été choisis après une période d'essais préliminaires. Ies figures I, II et III indiquent les manipulations opérées (changements de bains) et les courbes correspondantes obtenues.
\end{abstract}

La partie initiale de la congélation (de $+4^{\circ} \mathrm{C}$ au début de la cristallisation) est représentée 

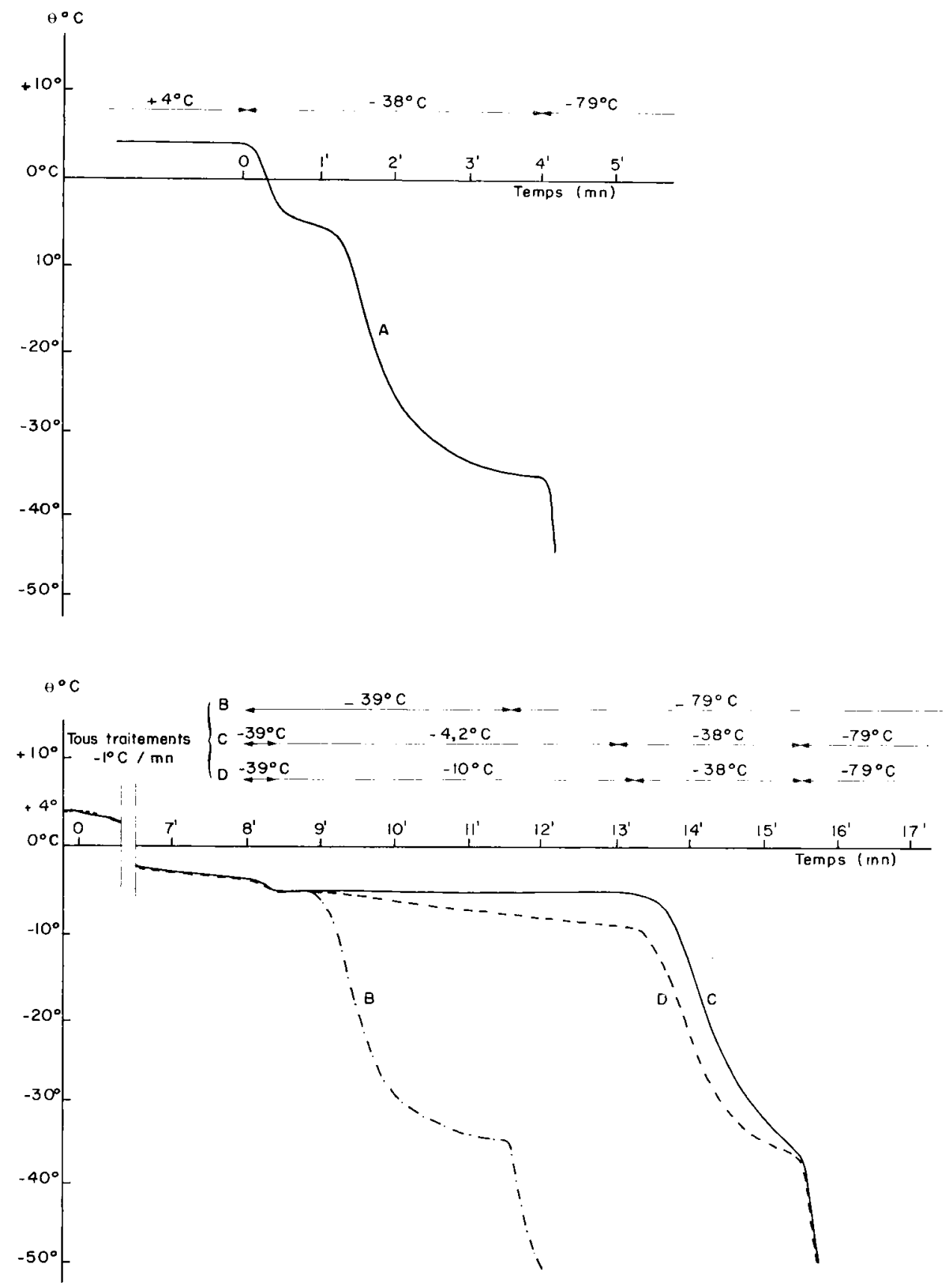

FIG. I, II, - Trailements A, B, C, D. Modes de refroidissement utilisés (temps de séjour des échantillons dans les bains d'alcool maintenus aux lempéraiures indiquées) et enregistrements correspondants (lhermocouples placés au cenire de l'échanitlon). 
dans la courbe $\Lambda$ par une chute brutale dont la pente est d'environ $24^{\circ} \mathrm{C} / \mathrm{mn}$. Les autres courbes montrent un abaissement de $\mathrm{I}^{\mathrm{O}} \mathrm{C} / \mathrm{mn}$, poursuivi juscuu'à $-3^{\circ} \mathrm{C}$ pour les traitements $\mathrm{B}, \mathrm{C}, \mathrm{D}$, et jusqu'à $-8^{\circ} \mathrm{C}$ pour les traitements $\mathrm{F}, \mathrm{F}$, ( , seuls à présenter la particularité de la surfusion et du réchauffement consécutif de l'ensemble de l'échantillon au moment de la formation des premiers cristaux.

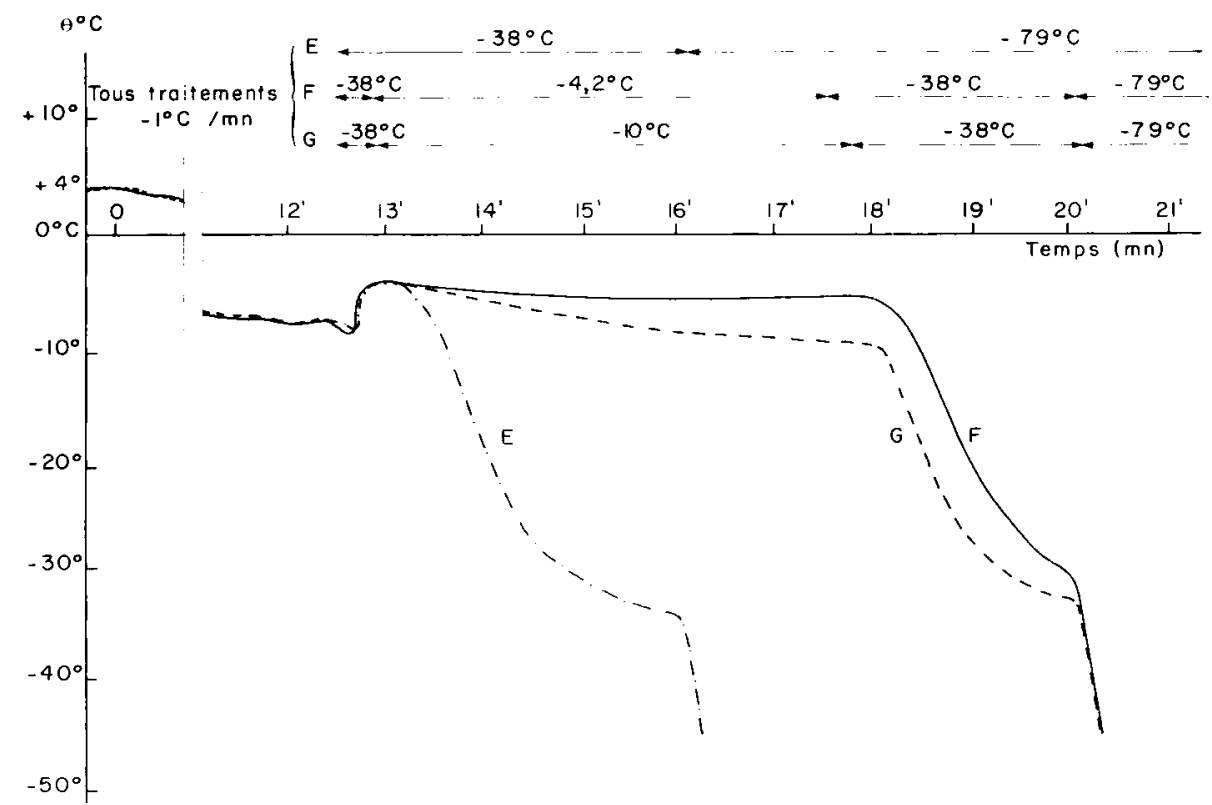

FIG. III. - Traitements E, F, G. Motes de refroidissement utilisés (lemps de séjour des échantillons dans les bains d'alcool maintenus aux températures indiquées) et enregistrements correspondants (thermo-couples placés au centre de l'échantillon).

Le plateau de début de cristallisition est réduit à 30 secondes pour les courbes $\mathrm{B}$ et $\mathrm{L}$ et fixé à 5 minutes pour les courbes $(,, F, 1)$ et $\mathrm{G}$. Il est horizontal et se maintient en moyenne à $--5^{\circ} \mathrm{C}$ pour $\mathrm{C}$ et $\mathrm{F}$ alors que les courbes $\mathrm{D}$ et ( $\mathrm{i}$ montrent pendant le même temps un abaissement moyen d'environ $0^{\circ} 8 \mathrm{C} / \mathrm{mn}$.

La partie terminale de la congélation est la mîme pour les 7 traitements : refroidissement de - ${ }^{\circ} 0^{\circ} \mathrm{C}$ à $-35^{\circ} \mathrm{C}$ en 2 minutes environ et de $35^{\circ} \mathrm{C}$ à $70^{\circ} \mathrm{C}$ en 40 secondes. $A$ près séjour de quelques minutes daus le bain ì -. $79^{\circ} \mathrm{C}$, les échantillons sont plongés dans l'azote liquide où ils sont conservés.

La scmence a été examinée après décongélation par immersion dans de l'ean à $+4^{\mathrm{o}} \mathrm{C}$, I ì 3 jours et 47 à 50 jours après congélation. Une codification des traitements et des taureaux, différente pour chaque jour de congélation, évite tout biais psychologique, sauf évidemment dans la détermination de l'effet de la durée de conservation. Chaque éjaculat a donné lieu à l'appréciation de I4 pourcentages de spermatozoïdes mobiles ultérieurement transformés en degrés d’arc, chacun de ces pourcentages représentant la moyenne des examens non consécutifs de 2 ampoules.

L'examen de la semence congelée selon les 7 diagrammes indi fués permet d'apprécier l'effet :

- du refroidissement initial (opposition entre A et B),

- du réchauffement ou plutôt de l'association surfusion-réchauffement au début de la cristallisation (opposition entre BCD et LFG),

- de la longueur et de l'inclinaison du plateau de début de cristallisation (opposition entre $\mathrm{BE}, \mathrm{CF}$ et $\mathrm{DG}$ ).

\section{RÉSUL'TATS}

Les caractères particuliers du diagramme $A$ n'ont pas permis de traiter les données relatives à cette courbe conjointement avec celles concernant 1'ensemble des autres courbes. Les comparaisons ont été limitées au seul diagramme B qui s'en 
rapproche le plus par la forme du plateau et qui en diffère surtout par la vitesse du refroidissement initial.

Les données moyennes relatives aux 2 diagrammes $\mathrm{A}$ et $\mathrm{B}$ et aux 2 durées de conservation considérées figurent dans le tableau $\mathrm{I}$. L'analyse de variance présentée dans le tableau 2 montre que, si la durée de conservation a une action certaine $(\mathrm{P}<\mathrm{o}, \mathrm{OI})$, la différence constatée entre les effets des refroidissements réalisés selon les diagrammes A et B n'est pas significative. Aucune interaction n'a été constatée entre le facteur taureau et les facteurs étudiés.

\section{TABLEAU I}

Pourcentages de spermazoïdes mobiles après décongélation du sperme refroidi selon les diagrammes $A$ et $B$

(données ayant subi la transformation angulaire)

\begin{tabular}{|c|c|c|c|c|c|}
\hline \multirow{2}{*}{ Taureaux } & \multicolumn{2}{|c|}{ Refroidissement initial } & \multicolumn{2}{|c|}{ Durée de conservation } & \multirow{2}{*}{ Moyennes } \\
\hline & A & B & 1 jour & 48 jours & \\
\hline 267 & 37,9 & 39,5 & $39,-\mathbf{x}$ & 37,9 & 38,6 \\
\hline 254 & $41,1_{4}$ & 40,2 & 41,9 & 39,8 & 40,8 \\
\hline 269 & 33,3 & 33,6 & $33, \mathbf{t}$ & 33,5 & 33,5 \\
\hline 259 & 40,6 & 41,5 & $41), 9$ & 41,3 & 41,1 \\
\hline 277 & 35,8 & 35, , $^{\prime}$ & 36,9 & 31,2 & 35,6 \\
\hline 830 & 37,4 & 38,8 & 38,9 & 37,2 & 38,1 \\
\hline Moyennes. & 37,7 & 38,2 & 38,6 & 37,3 & 37,9 \\
\hline & & & & & \\
\hline
\end{tabular}

TABLEAL 2

Analyse de variance limitée aux 4 combinaisons de traitements concernant les courbes $A$ et $B$ et les 2 temps de conservation de 1 et 48 jours

\begin{tabular}{|c|c|c|c|c|}
\hline Source de variation & $\begin{array}{l}\text { Somme } \\
\text { des carrés }\end{array}$ & D.L. & Carré moyen & $\mathrm{F}$ \\
\hline Taureaux et éjaculats.... & 4389,75 & 41 & 107,07 & $16,78 * *$ \\
\hline Courbe (A opposé à 13)... & 8,15 & 1 & 8,15 & $1,28 \mathrm{NS}$ \\
\hline Conservation ........ & 65,62 & 1 & 65,62 & $10,29 * *$ \\
\hline Courbe $\times$ Conservation. . & 3,72 & 1 & 3,72 & $0,58 \mathrm{NS}$ \\
\hline Erreur............ & 785,28 & 123 & 6,38 & \\
\hline Total & 5252,52 & 167 & & \\
\hline
\end{tabular}

Une factorielle $2 \times 3 \times 2$, excluant les données du traitement $A$, a permis d'étudier les effets du réchauffement au début de la cristallisation, de la longueur et de l'inclinaison du plateau qui suit cette cristallisation et de la durée de conser- 
vation de la semence. Les moyennes par taureau et traitement sont indiquées dans le tableau 3. L'analyse de variance (tabl. 4) montre que les 3 types de plateaux constituent une source hautement significative $(P<0,0 x)$ de variation du pourcentage de spermatozoïdes mobiles après congélation. L,e plateau $\mathrm{BE}$ de 30 secondes se révèle nettement supérieur aux 2 plateaux CF et I)G de 5 minutes $(\mathrm{P}<0,0 \mathrm{r})$.

\section{TABLEAU 3}

Pourcentage de spermatozoides mobiles après décongélation du sperme refroidi selon les diagrammes $B, C, D, E, F, G$,

(données ayant subi la transformation angulaire)

\begin{tabular}{|c|c|c|c|c|c|c|c|c|}
\hline \multirow[t]{2}{*}{ Taureaux } & \multicolumn{2}{|c|}{$\begin{array}{l}\text { Réchauffement } \\
\text { de } \\
\text { cristallisation }\end{array}$} & \multicolumn{3}{|c|}{ Type de plateau } & \multicolumn{2}{|c|}{$\begin{array}{c}\text { Durée } \\
\text { de } \\
\text { conservation }\end{array}$} & \multirow[t]{2}{*}{ Moyennes } \\
\hline & $\mathrm{BCD}$ & $\mathrm{EFG}$ & $\mathrm{BE}$ & $\mathrm{CF}$ & DG & 1 jour & 48 jours & \\
\hline 267 & 36,3 & 36,7 & 39,0 & 36,2 & 34,3 & 36,9 & 36,1 & 36,5 \\
\hline 254 & 37,2 & 39, ? $_{\mathrm{F}}$ & 41,0 & 37,4 & 36,5 & 39,3 & 37,2 & 38,3 \\
\hline 269 & 32,3 & 31,8 & 33,0 & 31,9 & 31,2 & 32,5 & 31,5 & 32,0 \\
\hline 259 & 39,5 & 37,8 & 40,3 & 37,9 & 37,6 & 38,9 & 38,3 & 38,6 \\
\hline 277 & 32,8 & 33,2 & 35,3 & 32,2 & 31,6 & 33,9 & 32,1 & 33,0 \\
\hline 830 & 36,3 & 36,9 & 38,6 & 36,5 & 31,7 & 37,3 & 36,0 & 36,6 \\
\hline Moyennes & 35,7 & 35,9 & 37,9 & 35,3 & 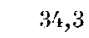 & 36,5 & 35,2 & 35,8 \\
\hline
\end{tabular}

\section{TABI,EAU 4}

Analyse de variance concernant les 12 combinaisons de traitements excluant la courbe $A$ (6 diagrammes de refroidissement : $\mathrm{B}, \mathrm{C}, \mathrm{D}, \mathrm{E}, \mathrm{F}, \mathrm{G}$, et 2 durées de conservation : I jour et 48 jours)

\begin{tabular}{|c|c|c|c|c|}
\hline Source de variation & $\begin{array}{l}\text { Somme } \\
\text { des } \\
\text { carrés }\end{array}$ & $\mathrm{DL}$ & $\begin{array}{l}\text { Carré } \\
\text { moyen }\end{array}$ & $\mathbf{F}$ \\
\hline 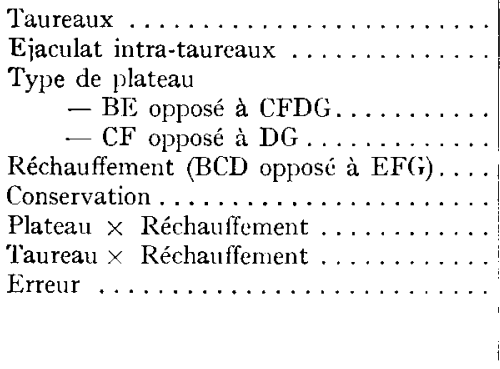 & $\begin{array}{r}3117,38 \\
8542,95 \\
1038,22 \\
93,2 \prime 1 \\
6,22 \\
203,17 \\
114,81 \\
181,61 \\
3232,3^{\prime} 1 \\
-2 . \\
16530,00\end{array}$ & $\begin{array}{r}5 \\
36 \\
1 \\
1 \\
1 \\
1 \\
2 \\
5 \\
51\end{array}$ & $\begin{array}{r}623,48 \\
237,30 \\
\\
038,22 \\
93,2 \text { t } \\
6,22 \\
203,17 \\
57,42 \\
36,33 \\
7,17\end{array}$ & $\begin{array}{c}86,99^{* *} \\
33,11^{* *} \\
144,87^{* *} \\
13,01^{* *} \\
0,87 \mathrm{NS} \\
28,35^{* *} \\
8,01^{* *} \\
5,07^{*}\end{array}$ \\
\hline
\end{tabular}

La différence hautement significative $(P<0,0 r)$, pour la survie des spermatozoïdes entre les 2 plateaux de pente différente CF et DG est essentiellement due à la différence entre $\mathrm{C}$ et $\mathrm{D}$, les traitements $\mathrm{F}$ et $\mathrm{G}$ ayant des effets analogues. $\mathrm{Du}$ fait de 
l'interaction plateau $\times$ réchauffement illustrée par la figure 4 , le réchauffement au début de la cristallisation, sans effet sur le sperme congelé selon les diagrammes $\mathrm{BE}$ ou $\mathrm{CF}$, paraît avoir nettement $(\mathrm{P}<\mathrm{o}, \mathrm{OI})$ favorisé le traitement $\mathrm{G}$ par rapport au traitement $\mathrm{D}$. L'effet défavorable de la conservation $(\mathrm{P}<0, \mathrm{oI})$ est réparti de façon homogène entre les différents niveaux des 2 autres facteurs expérimentés ainsi qu'entre les 6 taureaux utilisés. L'existence possible d'interactions secondaires et d'une interaction tertiaire n'a pas été examinée.

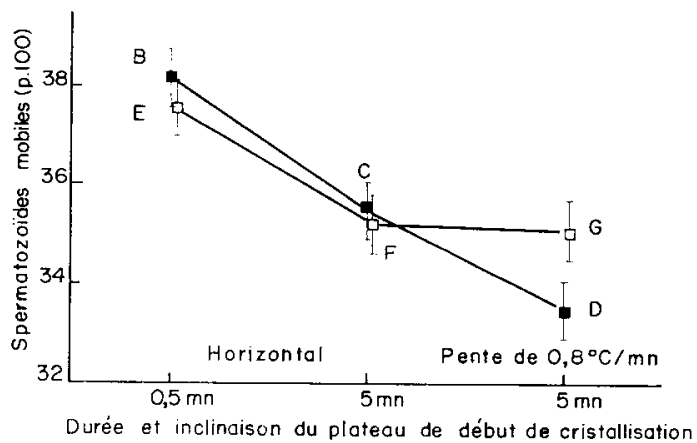

FIG. IV. - Pourcentages de spermatozoïdes mobiles après congélation pour les 6 traitements $B, C, D, E, F, G$. Interaction entre le type de plateau de début de crislallisation $(B E, C F, D G)$ el l'absence ou la présence de réchauffement au moment de la formation des premiers cristaux (BCD opposé à $E F G$ ).

Chaque point représente, pour un type de refroidissement donné, la moyenne de 84 pourcentages de spermatozoìdes mobiles ayant subi la transformation angulaire. L'intervalle de confiance s'entend au seuil de 0,05 .

$B, C, D$ : Absence de réchauffement au début de la cristallisation.

E, F, G : Présence d'un réchauffement au début de la cristallisation.

\section{DISCUSSION ET CONCLUSION}

Lorsqu'on refroidit de $\mathrm{I}^{\circ} \mathrm{C} / \mathrm{mn}$ des ampoules de verre de $\mathrm{I}, 2 \mathrm{ml}$ contenant $\mathrm{I} \mathrm{ml}$ de semence diluée dans le milieu glycérolisé utilisé dans cette expérimentation, les premiers cristaux de glace apparaissent à une température inférieure à celle du point normal de cristallisation $\left(-3,2^{\circ} \mathrm{C}\right)$. La surfusion se poursuit généralement au-dessous de $-8^{\circ} \mathrm{C}$ mais rarement au-dessous de $-\mathrm{I} 3^{\circ} \mathrm{C}$. Cette première formation de glace s'accompagne d'une production de chaleur (réchauffement) et entraîne l'augmentation de la concentration des substances dissoutes. Toute nouvelle formation de cristaux exige, non seulement 1'élimination de la chaleur produite par les cristallisations antérieures, mais une évacuation supplémentaire de calories pour que la température s'abaisse jusqu'au point normal de cristallisation relatif à la nouvelle concentration obtenue. On conçoit que puisse momentanément s'établir, grâce en partie à la très forte chaleur de fusion de l'eau, un équilibre entre production et dissipation de chaleur. Cet équilibre, variable selon les conditions du refroidissement est signalé par la persistance d'une température quasi-constante ou légèrement, décroissante (plateau de début de cristallisation).

$\mathrm{Si}$, au contraire, les échantillons sont refroidis plus rapidement (diagramme A), un gradient thermique important s'établit à l'intérieur du récipient (BRUEMmER 
et al., I963). Dans les ampoules utilisées, la surfusion et le réchauffement après cristallisation sont limités au film de liquide tapissant la paroi. A partir de cette couche, la cristallisation se propage partout où la température est inférieure à - $3,2^{\circ} \mathrm{C}$ $\mathrm{L}_{\text {a }}$ formation de la glace suit donc la progression du froid à l'intérieur de l'échantillon et débute successivement dans des zones se rapprochant graduellement de l'axe. La quantité de chaleur dégagée par la cristallisation pendant un instant donné est alors trop faible, eu égard à celle évacuée par conductivité, pour provoquer un réchauffement ou même un équilibre. La chute de température observée au centre de l'ampoule n'est que fortement ralentie et il ne se forme pas de plateau au sens strict.

On voit donc que le traitement $A$ diffère du traitement $B$ non seulement par la vitesse du refroidissement initial mais par un mode de cristallisation particulier. Aussi les conclusions défavorables à l'emploi d'un refroidissement brutal entre $+5^{\circ} \mathrm{C}$ et - IO ${ }^{\circ} \mathrm{C}$ (WILLE'T et SAIISBURY, I942; MCFEE, I959; KENNiLy, I960), ne sont pas expressément mises en cause par l'égalité des effets des traitements A et $B$. L'examen rigoureux de l'action de la vitesse du refroidissement initial paraîtobliger à l'emploi d'un mode de conditionnement différent de l'ampoule.

L'étude de l'influence du réchauffement et de la durée du plateau suppose la possibilité de déclencher à volonté une cristallisation simultanée dans la totalité de $1^{\prime}$ échantillon à $-3^{\circ} \mathrm{C}$ ou à $-8^{\circ} \mathrm{C}$. Iìn fait, cette cristallisation se produit de Io à I5 secondes après immersion de l'ampotle à -. $38^{\circ} \mathrm{C}$. L a surfusion des échantillons des traitements $B, E$, F est ainsi limitée au film liquide tapissant la paroi. L'étude des effets d'une surfusion plus profonde ainsi que la dissociation du couple surfusionréchauffement n'ont pu être réalisées avec le conditionnement décrit.

$I_{1}$ a différence entre les motilités relatives aux 2 ensembles de traitement $B, C$, $D$ et $E, F, G$ n'étant pas significative, l'apport de chaleur en profondeur, signalé par l'élévation thermique de quelques degrés constatée après surfusion, ne paraît pas avoir d'autre effet sur les spermatozoïles que celui d'un réchauffement quelconque opéré par conduction et convection.

I'endant la durée du plateau, çue la température clemeure à $-5^{\circ} \mathrm{C}(\mathrm{B}, \mathrm{E}, \mathrm{C}, \mathrm{F})$ ou qu'elle s'abaisse grarluellement jusqu'à $-8,5^{\circ} \mathrm{C}$ (1), G), les spermatozoüdes se trouvent dans une solution dont la tonicité, si on néglige les termes non linéaires de la loi de Raoult, est proportionnelle à la température négative enregistrée (I 60 p. Ioo de la tonicité initiale pour les plateaux horizontaux C, F et 200 p. Ioo en moyenne pour les plateaux inclinés I), G). Or SWANSON (I949), PURSLEY et Hermann (I950) ont constaté une diminution rapide du pourcentage de spermatozoïdes mobiles pour des éjaculats dilués et conservés à $+5^{\circ} \mathrm{C}$ dans des solutions de tonicité égale à I5o p.roo de la tonicité optimum. Il est donc possible que les différences observées entre les motilités relatives aux 3 types de plateaux étudiés soient liées au séjour plus ou moins long des cellules dans des solutions diversement concentrées où, en outre, le dépôt plus ou moins précoce et rapide de certains sels peut entrainer de fortes variations de pH (VAN I)EN BERG et Rose, r959).

Malgré $\mathrm{I}$ 'absence de différence entre $\mathrm{F}$ et $\mathrm{G}$ résultant de l'interaction inexpliquée entre durée du plateau et présence d'un réchauffement, les refroidissements lents $\left(0,8^{\circ} \mathrm{C} / \mathrm{mn}\right)$, consécutifs à la formation des premiers cristaux, paraissent au moins aussi nocifs que l'absence de tout refroidissement pendant le même temps.

Il est donc possible qu'une part des contradictions relevées dans la bibliographie 
à propos des vitesses de congélation résultent $\mathrm{du}$ fait que certains refroidissements lents ont été trop tardivement ou insuffisamment accélérés à partir du début de la cristallisation.

Ręu pour publication en octobre 1965.

\title{
SUMMARY
}

\author{
A STUDY ON THE EFFECTS (OF DIFFERENT METHODS OF COOLING \\ ON MOTILITY OF BULL SPERM WILN THAWED
}

42 ejaculates from 6 bulls were diluted in a skimmed milk-fructose-glycerol extender. Sealed up ampules containing the diluted semen were dipped into alcohol-baths at different temperatures so as to obtain different types of chilling.

The control samples were frozen according to a semirapid process of cooling, including neither supercooling nor rewarming nor standstill at the beginning of cristallization.

The other processes included a decresase of $\mathrm{I}^{\circ} \mathrm{C}$ per minute down to $-3^{\circ} \mathrm{C}$ without any supercooling and rewarming $(\mathrm{B}, \mathrm{C}, \mathrm{I})$ ) ; down to $-.8^{\circ} \mathrm{C}$ with supercooling and rewarming $(\mathrm{E}, \mathrm{F}, \mathrm{G}$.) After the starting of cristallization, a constant temperature was maintained for 30 seconds (E, B) or 5 minutes $(\mathrm{C}, \mathrm{F})$. $\mathrm{As}$ for $\mathrm{D}$ and $\mathrm{G}$ processes, a slow decrease $\left(0,8^{\circ} \mathrm{C}\right.$ per minute) was maintained for $5 \mathrm{mi}$ nutes. Lower than $10^{\circ} \mathrm{C}$, all samples were cooled at the same speed.

After thawing, samples A, B, E, were found to give the higher percentages of motile spermatozoa. The three of them did not significantly differ from one another. Likely results were obtained from samples B, C, D and E, F, G (whether supercooling, rewarming or not). (On another hand coupled treatments B, E; C, F $; \mathrm{D}$, G ; give different results. B, E give better results than $\mathrm{C}, \mathrm{F}$, which give better results than $\mathrm{D}, \mathrm{G}$.

These results suggest that the low values some authors found for slowly-cooled samples canbe related to delayed or unsufficient speeding up of the cooling process since the beginning of cristallization.

\section{RÉFÉRENCES BIBLIOGRAPHIQUES}

Bergfeld J., 1963 in vitro Tiefgefrierversuche $\left(-79^{\circ} \mathrm{C}\right)$ mit Bullensperma unter Anwendung von unterschiedlichen Verdünnungsmedien und Gefriergeschwindigkeiten. Zuchlhyg., 7. 319-325.

Bialy G.. Smith V. R., 1957. Rapid rates for freezing bull semen. J. Dairy Sci., 40, 622 (Abstr.).

Bruemmer J. H., Edpy R. W., Duryea W. J., ig63. Temperature control in the low temperature preservation of spermatozoa. J. Cell. Comp. Physiol., 62, I I 3-I 7 .

Goffaux M., 1963. Aptitude des taureaux à la production de semence capable de supporter la congélation. Ann. Zootech., 13, 87-92.

GoFfaux M., rg64. Influence de 2 caractères du processus de refroidissement du sperme de Taureau sur la motilité après congélation. Vih Intern. Congr. anim. reprod. ariff. insem. Trento IIIrd Secti, on687-692.

KenNely J. J. et al. Survival rates of rapidly frozen bovine spermatozoa. J. Dairy Sci., 43, r I40-r 146.

LOVELOCK J. E., I953. Cité par A. U. SMITH, I954. Effect of low temperature on living cells and tissues. In "Biological applications of freezing and drying" R. J. C. Harris. Acad. Press. Inc. Publ. New-York.

McFEE A. F., 1959. Effects of various cooling methods on spermatozoa livability. J. Dairy Sci. 42, 93 I (Abstr.).

Martig R. C., Dickey J. F., 1963. Freezability of bovine spermatozoa cooled rapidly with liquid nitrogen. J. Dairy Sci., 46, i 76 . (Abstr.)

Miller W. J., Van Demark N. L., I954. The influence of glycerol level, various temperature aspects and certain other factors on the survival of bull spermatozoa at sub-zero temperatures. J. Dairy Sci., $37,45-5$ I.

O'Deli, W. J., Almquist J. O., Marsh L. A., 1958. Freezing bovine semen III. Effect of freezing rate on bovine spermatozoa frozen and stored at - 79 C. J. Dairy Sci., 41, 79-89.

Pickett B. W., Martig R. C., Cowan W. A., ig6i. Preservation of bovine spermatozoa at - $79^{\circ} \mathrm{C}$ and - $19^{\circ} \mathrm{C}$. J. Dairy Sci., 44, $2089-2096$.

POLGE C.: Lovelock J. E., 1952. Preservation of Bull semen at $-79^{\circ} \mathrm{C}$. Veler. Rec., 64, 396, 397. 
l'olge C., 1957. Low temperature storage of mammalian spermatozoa. Proc. Roy. Soc., B 147, $498-507$. Polge C., Jakonsen F., 1959. Techniques for freezing bull semen. Veter. Rec., 71, 928-932.

Pursiey G. R., Hermans H. A., 1950. Some effects of hypertonic and hypotonic solutions on the livability and morphology of bovine spermatozoa. J. Dairy Sci., 33, 220-227.

Swanson E. W., I949. The effect of varying proportions of egg-yolk and sodium citrate buffer in bull semen diluters upon sperm motility. J. Dairy Sci., 32, 345-352.

Van den Berg L., Rose D., 1959. Cité dans A. U. S.Mith : Biological effecls of freezing and super cooling. $384-387$, E. Arnold Ltd - London, I96I.

Willett E. L., Salisbury G. W., 1942. Cité dans G. W. Salisbury and N. L. Van Demark. Physiology of reproduction and artificial insemination of callle, 388. W. H. Freeman and Co, San Francisco, 1961. 